\title{
SÍNDROME INFLAMATÓRIA PEDIÁTRICA MULTISISTÊMICA (PIMS) E A ASSOCIAÇÃO COM A SARS-COV-2
}

\author{
SÍNDROME INFLAMATORIO PEDIÁTRICO MULTISISTÉMICO (PIMS) Y LA \\ ASOCIACIÓN CON SARS-COV-2
}

\author{
MULTISISTEMIC PEDIATRIC INFLAMMATORY SYNDROME (PIMS) AND THE \\ ASSOCIATION WITH SARS-COV-2
}

Eduardo Haruo UCHIYA ${ }^{1}$

\begin{abstract}
RESUMO: Objetivo: Analisar a produção científica sobre a PIMS associado ao diagnóstico de SARS-CoV-2. Método: A revisão integrativa da literatura é o método empregado para a investigação. Foram adotados os seguintes critérios de inclusão: artigo original em inglês, disponível on-line e na íntegra: ano de publicação em 2020, na base de dados MEDLINE, no motor de busca PubMed. Os artigos que não responderem ao objetivo do estudo serão excluídos. Os descritores foram aplicados: Infecções por Coronavírus, Síndrome, Criança. Resultados: Cinco publicações foram incluídas e categorizadas em "Produção científica sobre a correlação da Síndrome inflamatória pediátrica com SARS-CoV-2". Mostrou nos principais resultados que, quatro estudos com crianças diagnosticadas com COVID-19 (recente ou anterior, com história passada) desenvolveram a síndrome e que um dos autores associa SARS-CoV-2 com a doença de Kawasaki. Conclusão: SARS-CoV-2 está relacionado à PIMS, independentemente do desenvolvimento da síndrome, pode gerar sérias complicações de saúde para a criança.
\end{abstract}

PALAVRAS-CHAVE: Infecções por coronavírus. Síndrome. Criança.

RESUMEN: Objetivo: Analizar la producción científica en PIMS asociada con el diagnóstico de SARS-CoV-2. Método: la revisión integradora de la literatura es el método utilizado para la investigación. Se adoptaron los siguientes criterios de inclusión: artículo original en inglés, disponible en línea y completo: año de publicación en 2020, en la base de datos MEDLINE, en el motor de búsqueda PubMed. Se excluirán los artículos que no respondan al objetivo del estudio. Se aplicaron los descriptores: Infecciones por coronavirus, Síndrome, Niño. Resultados: Se incluyeron y clasificaron cinco publicaciones en "Producción científica sobre la correlación del síndrome inflamatorio pediátrico con el SARS-CoV-2". En los resultados principales se mostró que cuatro estudios con niños diagnosticados con COVID-19 (reciente o anterior. Con antecedentes)) desarrollaron el síndrome y que uno de los autores asocia el SARS-CoV-2 con la enfermedad de Kawasaki Conclusión: El SARS-CoV-2 está relacionado con PIMS, independientemente del desarrollo del síndrome, puede generar complicaciones de salud graves para el niño.

PALABRAS CLAVE: Infecciones por coronavirus. Síndrome. Infantil.

${ }^{1}$ Centro Universitário das Américas (FAM), São Paulo - SP - Brasil. Discente do Curso em Bacharel de Enfermagem. ORCID: https://orcid.org/0000-0003-2942-7641. E-mail: eduardoharuouchiya@ hotmail.com

Temas em Educ. e Saúde, Araraquara, v. 16, n. 1, p. 9-20, jan./jun., 2020. e-ISSN 2526-3471. 
ABSTRACT: Introduction: PIMS means pediatric inflammatory multisystemic syndrome. It describes a new health condition seen in children who were infected with new coronaviruses, recovered and later show an immune response that results in significant levels of inflammation in the organ systems and symptoms. Objective: To analyze scientific production on Systemic Pediatric Inflammatory Syndrome (PIMS) in relation to the diagnosis of SARS-CoV-2. Method: The integrative literature review is the method employed for investigation. The following inclusion criteria were adopted: original article in English, available online and in full: year of publication in 2020, in the MEDLINE database, on the PubMed Search Engine. Articles that do not respond to the objective of the study will be excluded. The descriptors applied were: Coronavirus Infections, Syndrome, Child. Results: Five publications were included and categorized in "Scientific production on the correlation of Pediatric Inflammatory Syndrome with SARS-CoV-2". It showed in the main results that in the analysis of the studies, four studies of children diagnosed with COVID-19 (recent or previous, with past history) developed the syndrome, and that one of the authors links SARS-CoV-2 with Kawasaki Disease (DK) with disagreement. Conclusion: SARS-CoV-2 is related to PIMS, regardless of the development of the syndrome, it can generate serious health complications for the child.

KEYWORDS: Coronavirus infections. Syndrome. Child.

\section{Introdução}

Desde o início da pandemia de COVID-19, as crianças eram uma população relativamente intocada pelo novo corona vírus. Se infectadas, as crianças geralmente apresentavam poucos sintomas, a menos que uma condição subjacente comprometesse sua saúde (CHILDREN'S HOSPITAL LOS ANGELES, 2020).

A maioria das crianças com infecção por SARS-CoV-2 é assintomática ou apresenta sintomas leves da infecção. Até o momento, as crianças responderam por uma porção mínima dos casos na pandemia global da COVID-19 e dados epidemiológicos de muitos países mostram que são minoria entre os pacientes afetados. Crianças e adolescentes menores de 18 anos representaram apenas $1,7 \%$ dos casos reportados nos EUA, $1 \%$ na Holanda e $2 \%$ de uma grande coorte observacional no Reino Unido. Estudos de vários países confirmaram que doenças graves e morte por COVID-19 em crianças são raras, apesar de baixa precisão dos dados devido à ausência de verdadeiros denominadores populacionais. Entretanto, agora a atenção aumentou em relação à vulnerabilidade das crianças, por dois motivos: em primeiro lugar, o conhecimento do grau em que as crianças transmitem COVID-19 é fundamental para se saber como os países reabrirão as comunidades após o bloqueio e, em segundo lugar, nos últimos dois meses foram identificadas crianças que desenvolveram uma resposta inflamatória sistêmica significativa - devido a isso estão ocorrendo diversas descrições de novas manifestações de uma doença grave, semelhante à doença de Kawasaki, relacionada à infecção por COVID-19, trazendo uma nova faceta dessa doença (BEREZIN, 2020). 
Algumas crianças apresentaram algumas ou todas as características observadas na doença de Kawasaki, uma doença de crianças que pode resultar em aumento ou aneurisma das artérias coronárias. As características observadas incluíram febre, erupção cutânea, conjuntivite; mãos vermelhas e inchadas; e lábios vermelhos e rachados. Algumas crianças apresentaram sinais clínicos e laboratoriais da síndrome da tempestade com citocinas, uma resposta imune sistêmica exagerada que causou danos aos órgãos em adultos com COVID-19 (BOSTON CHILDREN'S HOSPITAL, 2020).

PIMS significa síndrome multissistêmica inflamatória pediátrica. Ele descreve uma nova condição de saúde observada em crianças que foram infectadas com novos coronavírus, recuperadas e mais tarde apresentam uma resposta imune que resulta em níveis significativos de inflamação nos sistemas e sintomas de órgãos. O PIMS é semelhante a outras condições inflamatórias, como a doença de Kawasaki e a síndrome do choque tóxico. As crianças que têm PIMS geralmente não apresentaram sintomas óbvios quando infectadas com novos coronavírus, como tosse, e geralmente eram saudáveis antes do desenvolvimento de PIMS (CHILDREN'S HOSPITAL LOS ANGELES, 2020).

Finalmente, muitas crianças tiveram coagulopatias; disfunção cardíaca; diarreia, distensão abdominal e outros sintomas gastrointestinais (com algumas crianças tendo testes de fezes positivos para SARS-CoV-2); ou lesão renal aguda. Os sintomas respiratórios nem sempre foram uma característica proeminente (BOSTON CHILDREN'S HOSPITAL, 2020).

As informações disponíveis sobre o PIMS são extremamente limitadas devido ao pequeno número de casos em comparação com o número impressionante de pessoas afetadas pelo COVID-19 (CHILDREN'S HOSPITAL LOS ANGELES, 2020). Nesse sentido, o presente tem como objetivo analisar produções cientificas que tratem sobre PIMS em relação com o diagnóstico de SARS-CoV-2.

\section{Método}

Este estudo utiliza-se do método de levantamento bibliográfico, a partir da revisão integrativa de literatura, que tem como função sintetizar resultados de estudos anteriores sobre o assunto proposto.

As revisões integrativas têm o potencial de evidenciar compreensão abrangente sobre assuntos específicos e apontar lacunas existentes no conhecimento. As etapas seguidas na elaboração desta revisão serão: estabelecimento da questão da pesquisa, busca na literatura, avaliação dos dados, análise dos estudos incluídos, interpretação dos resultados e apresentação da revisão (SOUZA; SILVA; CARVALHO, 2010). 
A coleta de dados foi realizada em junho de 2020, na base de dados Medical Literature Analysis and Retrieval System Online (MEDLINE), no motor de busca da Biblioteca Nacional de Medicina dos Estados Unidos (PubMed) e teve critérios de inclusão, artigos originais, na língua inglesa, disponíveis online e na integra, com ano de publicação de 2020, e que respondam a questão: "Qual é a relação da COVID-19 com a Síndrome Inflamatória Pediátrica Sistêmica (PIMS)?"

Foram aplicados os Descritores em Saúde (DeCS): Infecções por Coronavírus, Síndrome, Criança, bem como suas respectivas versões em inglês e espanhol.

Com o objetivo de delimitar a pesquisa, foram excluídas publicações com os assuntos principais: Síndrome periódica associada à criopirina (CAPS), NLRC4, Meningite, Vírus da Imunodeficiência Humana (HIV), Eosinofilia, Sarcoidose pediátrica, Mucopolissacaridose tipo II, por não se tratar do assunto desta revisão.

A identificação dos artigos foi feita de forma independente, total de cinquenta e quatro artigos completos disponíveis. Foram excluídos trabalhos como teses, títulos e resumos que não correspondem a proposta da revisão,

No período maio para junho de 2020, com uma leitura detalhada dos artigos, foi realizada a análise de dados.

Para o desenvolvimento da análise, foi elaborado um formulário com os seguintes itens: título, autores, ano, base de dados, país de origem e nível de evidência.

Para a categorização do nível de evidência, foram considerados sete níveis de classificação: nível 1, revisão sistemática, ou metanálise de ensaios clínicos controlados; nível 2, ensaio clínico controlado randomizado bem delineado; nível 3, ensaio clínico controlado sem randomização; nível 4, estudos de coorte ou caso-controle bem delineados; nível 5, revisão sistemática de estudos qualitativos e descritivos; nível 6, estudos descritivos ou qualitativos e nível 7, opinião de autoridades e especialistas (GALVÃO, 2006).

O paper por se tratar de uma revisão não houve necessidade de parecer do Comitê de Ética.

\section{Resultados}

Entre cinquenta e quatro artigos selecionados na busca de dados na integra, entre maio e junho de 2020, somente cinco publicações foram incluídas no estudo na base de dados Medical Literature Analysis and Retrieval System Online (MEDLINE), no motor de busca da 
Biblioteca Nacional de Medicina dos Estados Unidos (PubMed), são apresentadas no Quadro 1.

Quadro 1 - Quantitativo de publicações encontradas nas bases de dados e incluídas no estudo

\begin{tabular}{|l|l|l|l|}
\hline Base & Motor de busca & Encontrada & Incluídas \\
\hline MEDLINE & PubMed & 54 & 5 \\
\hline
\end{tabular}

Fonte: elaborado pelo autor.

O Quadro 2 mostra a caracterização dos artigos, considerando também o nível de evidência. Os cinco artigos incluídos na revisão, foram publicados em língua inglesa, no ano de 2020. Na maioria, as pesquisas foram realizadas nos Estados Unidos $(n=3)$.

Quadro 2 - Caracterização das publicações e níveis de evidência dos artigos incluídos na revisão.

\begin{tabular}{|c|c|c|c|c|c|c|}
\hline $\mathbf{N}^{\circ}$ & Título & Autores & Ano & Revista & País & $\begin{array}{l}\text { Nível de } \\
\text { Evidência }\end{array}$ \\
\hline $1^{6}$ & $\begin{array}{l}\text { Acute heart } \\
\text { failure in } \\
\text { multisystem } \\
\text { inflammatory } \\
\text { syndrome in } \\
\text { children } \\
\text { (MIS-C) in the } \\
\text { context of global } \\
\text { SARS-CoV-2 } \\
\text { pandemic. }\end{array}$ & $\begin{array}{l}\text { Belhadjer, Z.; } \\
\text { Méot, M.; } \\
\text { Bajolle, F.; } \\
\text { Khraiche, D.; } \\
\text { Legendre, A.; } \\
\text { Abakka, S.; } \\
\text { Auriau, J.; } \\
\text { Grimaud, M.; } \\
\text { Oualha, M.; } \\
\text { Maurice, B.; } \\
\text { Wacker, J.; } \\
\text { Ovaert, C.; } \\
\text { Hascoet, S.; } \\
\text { Selegny, M.; } \\
\text { Malekzadeh- } \\
\text { Milani, S.; } \\
\text { Maltret, A.; } \\
\text { Bosser, G.; } \\
\text { Giroux, N.; } \\
\text { Bonnemains, } \\
\text { L.; } \\
\text { Bordet, J.; } \\
\text { Filippo, S.; } \\
\text { Mauran, P.; } \\
\text { Falcon-Eicher, } \\
\text { S.; } \\
\text { Thambo, J.; } \\
\text { Lefort, B.; } \\
\text { Moceri, P.; } \\
\text { Houyel, L.; } \\
\text { Renolleau, S.; } \\
\text { Bonne, D. }\end{array}$ & 2020 & CIRCULATION & França & 6 \\
\hline
\end{tabular}

Temas em Educ. e Saúde, Araraquara, v. 16, n. 1, p. 9-20, jan./jun., 2020. e-ISSN 2526-3471. 


\begin{tabular}{|c|c|c|c|c|c|c|}
\hline $2^{7}$ & $\begin{array}{l}\text { Pediatric Crohn's } \\
\text { disease and } \\
\text { multisystem } \\
\text { inflammatory } \\
\text { syndrome in } \\
\text { children (MIS-C) } \\
\text { and COVID-19 } \\
\text { treated with } \\
\text { infliximab }\end{array}$ & $\begin{array}{l}\text { Dolinger, M.; } \\
\text { T; Person, H.; } \\
\text { Smith, R.; } \\
\text { Jarchin, L.; } \\
\text { Pittman, N.; } \\
\text { Dubinsky, M. } \\
\text { C.; } \\
\text { Lai, J. }\end{array}$ & 2020 & $\begin{array}{l}\text { Journal Pediatric } \\
\text { Gastroenterology } \\
\text { and Nutrition }\end{array}$ & $\begin{array}{l}\text { Estados } \\
\text { Unidos }\end{array}$ & 6 \\
\hline $3^{8}$ & $\begin{array}{l}\text { COVID-19- } \\
\text { Associated } \\
\text { Pediatric } \\
\text { Multisystem } \\
\text { Inflammatory } \\
\text { Syndrome }\end{array}$ & $\begin{array}{l}\text { Leon, M. P. D.; } \\
\text { Redzepi, A.; } \\
\text { McGrath, E.; } \\
\text { Abdel-Haq, N.; } \\
\text { Shawaqfeh, A.; } \\
\text { Sethuraman, U.; } \\
\text { Tilford, B.; } \\
\text { Chopra, T.; } \\
\text { Arora, H.; } \\
\text { Ang, J.; } \\
\text { Asmar, B. }\end{array}$ & 2020 & $\begin{array}{l}\text { Journal of the } \\
\text { Pediatric } \\
\text { Infectious } \\
\text { Diseases Society }\end{array}$ & $\begin{array}{l}\text { Estados } \\
\text { Unidos }\end{array}$ & 6 \\
\hline $4^{9}$ & $\begin{array}{l}\text { Pediatric } \\
\text { Coronavirus } \\
\text { Disease-2019- } \\
\text { Associated } \\
\text { Multisystem } \\
\text { Inflammatory } \\
\text { Syndrome }\end{array}$ & Shulman, T, S. & 2020 & $\begin{array}{l}\text { Journal of the } \\
\text { Pediatric } \\
\text { Infectious } \\
\text { Diseases Society }\end{array}$ & $\begin{array}{l}\text { Estados } \\
\text { Unidos }\end{array}$ & 6 \\
\hline $5^{10}$ & $\begin{array}{l}\text { Multisystem } \\
\text { Inflammatory } \\
\text { Syndrome with } \\
\text { Features of } \\
\text { Atypical } \\
\text { Kawasaki Disease } \\
\text { during COVID-19 } \\
\text { Pandemic }\end{array}$ & $\begin{array}{l}\text { Rauf, A.; } \\
\text { Vijayan, A.; } \\
\text { John, S. T.; } \\
\text { Krishnan, R.; } \\
\text { Latheef, A. }\end{array}$ & 2020 & $\begin{array}{l}\text { The Indian } \\
\text { Journal of } \\
\text { Pediatrics }\end{array}$ & Índia & 6 \\
\hline
\end{tabular}

Fonte: elaborado pelo autor

A análise de dados está demonstrada na figura 3, que aponta os resultados dos estudos que permitiram a classificação em apenas uma categoria: "Produção científica sobre a correlação do Síndrome Inflamatória Pediátrica com SARS-CoV-2”.

\section{Produção científica sobre a correlação do Síndrome Inflamatória Pediátrica com SARS- CoV-2}

Dentre os artigos que apontam a associação da SARS-CoV-2 com a Síndrome Inflamatória Pediátrica, cinco artigos publicados, majoritariamente, em periódicos de medicina foram classificados nessa categoria. O Quadro 3, descreve resumidamente cada um dos estudos, abordando desenho, amostra populacional e principais resultados. 
Quadro 3 - Desenho, amostra populacional e principais resultados dos estudos.

\begin{tabular}{|c|c|c|c|c|}
\hline $\mathrm{N}^{\circ}$ & Desenho & Objetivo & $\begin{array}{l}\text { Amostra } \\
\text { Populacional }\end{array}$ & Principais Resultados \\
\hline $1^{6}$ & Descritivo & $\begin{array}{l}\text { Descrever uma } \\
\text { nova síndrome } \\
\text { complexa em } 35 \\
\text { crianças } \\
\text { internadas por } \\
\text { cardiopatia aguda }\end{array}$ & Crianças $\mathrm{N}=35$. & $\begin{array}{l}\text { Trinta e cinco pacientes } \\
\text { preencheram os critérios de } \\
\text { inclusão: choque cardiogênico } \\
\text { febril ou disfunção ventricular } \\
\text { esquerda e estado inflamatório. } \\
\text { A infecção por SARS-Cov- } 2 \text { foi } \\
\text { confirmada em } 31 / 35 \text { pacientes } \\
(88,5 \%) \text {. Swab nasofaríngeo } \\
\text { reação em cadeia da polimerase } \\
\text { (PCR) foi positiva em } 12 \text { pacientes } \\
\text { (34\%) e PCR fecal em } 2 \\
\text { pacientes (6\%). Trinta e trinta e } \\
\text { cinco ( } 86 \%) \text { pacientes tiveram } \\
\text { ensaios positivos de anticorpos: } 23 \\
\text { tiveram IgA e IgG, } 3 \text { tinham IgG, } 2 \\
\text { tinham IgG e IgM e } 2 \text { tinham } \\
\text { apenas IgA. Além disso, dois } \\
\text { pacientes eram negativos para PCR } \\
\text { SARS-CoV-2, mas apresentavam } \\
\text { características típicas de } \\
\text { tomografia de pulmão da } \\
\text { pneumonia por COVID. } \\
\text { Todos os pacientes apresentaram } \\
\text { estado inflamatório grave, } \\
\text { evidenciado por elevação de C- } \\
\text { reativa. }\end{array}$ \\
\hline $2^{7}$ & Descritivo & $\begin{array}{l}\text { Descrever um } \\
\text { caso de infecção } \\
\text { grave por } \\
\text { COVID-19 em } \\
\text { um paciente } \\
\text { pediátrico } \\
\text { recentemente } \\
\text { diagnosticado } \\
\text { com doença de } \\
\text { Crohn. }\end{array}$ & Criança $\mathrm{N}=1$. & $\begin{array}{l}\text { Os autores declaram que é o } \\
\text { primeiro caso de doença de Crohn } \\
\text { recentemente diagnosticada } \\
\text { com suspeita de síndrome } \\
\text { inflamatória multissistêmica em } \\
\text { crianças (MIS-C) relacionada } \\
\text { temporalmente ao COVID-19. } \\
\text { Segundo os autores, a tempestade } \\
\text { de citocinas foi considerada uma } \\
\text { principal causa de morbidade em } \\
\text { pacientes com infecção grave por } \\
\text { COVID-19. }\end{array}$ \\
\hline $3^{8}$ & Descritivo & $\begin{array}{l}\text { Descrever um } \\
\text { caso de possível } \\
\text { vínculo da doença } \\
\text { de coronavírus } \\
2019 \text { (COVID- } \\
\text { 19) com uma } \\
\text { doença } \\
\text { semelhante a } \\
\text { Kawasaki e } \\
\text { choque em } \\
\text { Europa. }\end{array}$ & Criança $\mathrm{N}=1$. & $\begin{array}{l}\text { A infecção por COVID-19 em } \\
\text { crianças foi tipicamente mais } \\
\text { suave do que em adultos. } \\
\text { Apresentações pediátricas do } \\
\text { COVID-19 foram diversos; no } \\
\text { entanto, pouco frequentemente } \\
\text { casos graves foram relatados. } \\
\text { A ausência de apresentações } \\
\text { semelhantes ao nosso caso em } \\
\text { publicações recentes na China } \\
\text { pode indicar uma predisposição } \\
\text { genética para complicações }\end{array}$ \\
\hline
\end{tabular}

Temas em Educ. e Saúde, Araraquara, v. 16, n. 1, p. 9-20, jan./jun., 2020. e-ISSN 2526-3471. 


\begin{tabular}{|c|c|c|c|c|}
\hline & & & & $\begin{array}{l}\text { cardíacas ou um quadro } \\
\text { inflamatório previamente não } \\
\text { reconhecido como resposta ao } \\
\text { COVID-19. Instamos um aumento } \\
\text { da vigilância de doenças } \\
\text { cardiovasculares complicações, } \\
\text { incluindo doença semelhante à } \\
\text { Doença de Kawasaki (KD), } \\
\text { miocardite e choque febris nas } \\
\text { crianças com COVID-19. }\end{array}$ \\
\hline $4^{9}$ & Descritivo & $\begin{array}{l}\text { Descrever e } \\
\text { caracterizar casos } \\
\text { propostos da } \\
\text { doença de } \\
\text { coronavírus-2019 } \\
\text { (COVID-19) } \\
\text { associada a } \\
\text { síndrome, } \\
\text { inflamatória } \\
\text { pediátrica } \\
\text { multissistêmica } \\
\text { (MSI-C). }\end{array}$ & $\begin{array}{l}\text { Crianças } \cong 100 \mathrm{em} \\
\text { Nova York, } 8 \mathrm{em} \\
\text { Londres, } 10 \mathrm{na} \\
\text { Itália, } 6 \mathrm{em} \\
\text { Filadélfia. }\end{array}$ & $\begin{array}{l}\text { Nem todos apresentaram resultado } \\
\text { positivo em ensaio de reação em } \\
\text { cadeia da polimerase (PCR) } \\
\text { e/ou imunoglobulina (Ig) G e/ou } \\
\text { Anticorpo IgM para graves } \\
\text { respiratórios síndrome coronavírus } \\
2 \text { (SARS-CoV-2). } \\
\text { Estudos de laboratório incluíram } \\
\text { marcadores de inflamação, } \\
\text { particularmente D-dímeros } \\
\text { elevados, ferritina e triglicerídeos } \\
\text { (altamente sugestivo de } \\
\text { macrófagos de síndrome de } \\
\text { ativação [MAS]), bem como } \\
\text { enzimas cardíacas e troponina } \\
\text { muito elevadas e extremamente } \\
\text { alto N-terminal-pro } \\
\text { Peptídeo natriuético do tipo B } \\
\text { (NT-pro-BNP) } \\
\text { Temporalmente, essas doenças } \\
\text { começaram a se manifestar } \\
\text { aproximadamente } 1 \text { mês ou } \\
\text { mais após o pico de casos COVID- } \\
\text { 19 em sua região, em vez de } \\
\text { contemporaneamente } \\
\text { com o pico nestas áreas fortemente } \\
\text { impactadas. Curiosamente, } \\
\text { crianças com esta síndrome não } \\
\text { foram descritas nas primeiras } \\
\text { séries de casos pediátricos em } \\
\text { localizações hiperepidêmicas de } \\
\text { SARS-2 na China e Itália. } \\
\text { Surpreendentemente, não há } \\
\text { evidências de que a síndrome } \\
\text { inflamatória multissistêmica } \\
\text { (MIS-C) tenha ocorrido em } \\
\text { crianças na Ásia. } \\
\text { As características clínicas do MIS- } \\
\text { C incluem dor abdominal muito } \\
\text { mais impressionante, diarreia, } \\
\text { vômito e envolvimento de vários } \\
\text { órgãos, } \\
\text { incluindo lesão renal aguda, }\end{array}$ \\
\hline
\end{tabular}

Temas em Educ. e Saúde, Araraquara, v. 16, n. 1, p. 9-20, jan./jun., 2020. e-ISSN 2526-3471. 


\begin{tabular}{|c|c|c|c|c|}
\hline & & & & $\begin{array}{l}\text { e relativamente poucos critérios } \\
\text { clássicos de KD quando } \\
\text { comparado com crianças com DK. } \\
\text { Características cardíacas do MIS-C } \\
\text { de maneira mais dramática } \\
\text { mostram moderado a muito grave } \\
\text { o envolvimento miocárdico } \\
\text { (manifestado por imagem e } \\
\text { surpreendentemente alto NT-pro- } \\
\text { BNP níveis de troponina), muito } \\
\text { maiores que associada à síndrome } \\
\text { de choque KD ou KD. }\end{array}$ \\
\hline $5^{10}$ & Descritivo & $\begin{array}{l}\text { Descrever } \\
\text { síndrome } \\
\text { inflamatória com } \\
\text { características } \\
\text { clínicas } \\
\text { simulando a } \\
\text { doença de } \\
\text { Kawasaki, } \\
\text { durante a } \\
\text { pandemia de } \\
\text { COVID-19 }\end{array}$ & Criança $\mathrm{N}=1$. & $\begin{array}{l}\text { As características do laboratório } \\
\text { foram neutrofilia, PCR elevada e } \\
\text { características clínicas incluindo } \\
\text { dor abdominal, sintomas } \\
\text { gastrointestinais, miocardite } \\
\text { e choque. } \\
\text { O Diagnóstico de KD “atípico" } \\
\text { realizado em pacientes que } \\
\text { apresentam características clínicas } \\
\text { atípicas ou incomuns. Embora } \\
\text { algumas evidências de miocardite } \\
\text { tenham sido observadas em mais } \\
\text { de } 50 \% \text { dos pacientes na fase } \\
\text { aguda da DK, miocardite } \\
\text { sintomática com choque } \\
\text { cardiogênico é raro. } \\
\text { A infecção por COVID e KD não } \\
\text { está comprovada conclusivamente. } \\
\text { No entanto, como relatórios } \\
\text { semelhantes vieram de vários } \\
\text { países com relação temporal com a } \\
\text { infecção por COVID na } \\
\text { comunidade, pediatras em geral } \\
\text { precisam estar cientes de tais } \\
\text { apresentações atípicas e realizar } \\
\text { encaminhamento precoce para } \\
\text { cuidados terciários. }\end{array}$ \\
\hline
\end{tabular}

Fonte: elaborado pelo autor

A Síndrome Inflamatória Pediátrica Multissistêmicas (PIMS) é descrita pelos autores como uma síndrome semelhante com a Doença de Kawasaki (KD), com exceção de apenas um estudo, que caracteriza a síndrome como diagnóstico principal (RAUF et al., 2020).

Um estudo contradiz o diagnóstico da Doença de Kawasaki (KD), devido à as características clínicas do MIS-C que incluem dor abdominal muito mais impressionante, diarreia, vômito e envolvimento de vários órgãos, incluindo lesão renal aguda, níveis alto de troponina (NT-pro-BNP), muito maiores que associada à síndrome de choque KD ou KD, o que caracteriza poucos critérios clássicos de KD (SHULMAN, 2020). 
Todos estes foram estudos descritivos de casos e remetem que a patologia associada a COVID-19 (diagnóstico recente ou histórico anterior), gera choque cardiogênico febril ou disfunção ventricular esquerda, miocardite, PCR elevadas e estado inflamatório graves com produção de marcadores inflamatórios.

Segundo um dos autores, temporalmente a doença, começara a manifestar aproximadamente 1 mês ou mais após o pico de casos COVID-19, entretanto, as informações disponíveis na literatura sobre essa teoria são insuficientes (SHULMAN, 2020).

Deve-se atentar a predisposição genética para complicações cardíacas ou um inflamatório previamente não reconhecido resposta ao COVID-19, é de suma importância o aumento da vigilância (LEON, 2020), mesmo com perfil epidemiológico baixo, sua gravidade é inquestionável (SHULMAN, 2020).

\section{Discussão}

Pensa-se que a infecção por SARS-CoV-2 em crianças seja relativamente leve em comparação com pacientes adultos e frequentemente assintomática ou minimamente sintomática. Até o momento, é limitado o conhecimento de possível lesão cardiovascular relacionada à SARS-CoV-2 em pacientes pediátricos população. No entanto, recentemente observa-se um número inesperadamente grande de crianças internados em unidades de terapia intensiva por choque cardiogênico ou disfunção aguda do ventrículo esquerdo no cenário de um estado inflamatório multissistêmico, com uma grande proporção dos que testaram apresentando resultado positivo para SARS-CoV-2 (BELHADJER et al., 2020).

Relatos de casos e pequenas séries descreveram uma apresentação de doença aguda acompanhada de uma síndrome hiperinflamatória, levando a falência e choque de múltiplos órgãos. As hipóteses iniciais são de que essa síndrome pode estar relacionada ao COVID-19 com base em testes laboratoriais iniciais que mostram sorologia positiva em maioria dos pacientes (WHO, 2020).

Essa rara síndrome que acomete crianças compartilha características comuns com outras condições inflamatórias pediátricas, incluindo: doença de Kawasaki, síndromes de choque tóxicos estafilocócicas e estreptocócicas, sepse bacteriana e síndromes de ativação de macrófagos. Também pode apresentar sintomas abdominais incomuns com marcadores inflamatórios excessivos (DAIC, 2020). 
Inúmeras questões são levantadas pelo reconhecimento do muito novo MIS-C. Estes relacionam-se à sua definição, patogênese, epidemiologia, genética, suscetibilidade, diagnóstico, terapia e sequelas e outros. A jornada está apenas começando (SHULMAN, 2020).

Existe, portanto, uma necessidade urgente de coleta de dados padronizados que descrevam apresentações clínicas, gravidade, resultados e epidemiologia (WHO, 2020).

\section{Conclusão}

O estudo evidenciou que a análise da produção científica sobre Síndrome Inflamatória Pediátrica Multissistêmica demostra a possibilidade de a síndrome estar relacionada com a SARS-CoV-2, o que a diferencia da Doença de Kawasaki por inúmeros determinantes.

Existe uma certa discordância sobre o tempo de janela imunológica para o desenvolvimento da síndrome, entretanto, é aceito que crianças (com infecção recente ou que possuíram histórico anterior do Diagnóstico de COVID-19) tiveram o desenvolvimento da patologia.

\section{REFERÊNCIAS}

BELHADJER, Z. et al. Acute heart failure in multisystem inflammatory syndrome in children (MIS-C) in the context of global SARS-CoV-2 pandemic. Circulation, Paris, 2020. Disponível em: https://www.ahajournals.org/doi/10.1161/CIRCULATIONAHA.120.048360. Acesso em: maio 2020.

BOSTON CHILDREN'S HOSPITAL. COVID-19 and a serious inflammatory syndrome in children: unpacking recent warnings. Boston,2020.

DAIC. Delivering news, technologies, trends on cath labs across the country. Kawasaki-like inflammatory disease affects children with COVID-19. [S. 1.], 2020.

DOLINGER, M. T. et al. Pediatric Crohn's disease and multisystem inflammatory syndrome in children (MIS-C) and COVID-19 treated with infliximab. Journal Pediatric Gastroenterology and Nutrition, New York, 2020. Disponível em: https://journals.lww.com/jpgn/Abstract/9000/Pediatric_Crohn_s_Disease_and_Multisystem.9 6044.aspx. Acesso em: maio 2020.

LEON, M. P. D et al. COVID-19-Associated pediatric multisystem inflammatory syndrome. Journal of the Pediatric Infectious Diseases Society, Michigan, 2020.

LOS ANGELES CHIELDREN'S HOSPITAL. Multisystem inflammatory syndrome in children (MIS-C): what parents should know. Los Angeles, 2020. 
RAULF, A. et al. Multisystem inflammatory syndrome with features of atypical kawasaki disease during COVID-19 pandemic. The Indian Journal of Pediatrics, Kerala, 2020.

SHULMAN, S. T. et al. Pediatric coronavirus disease-2019-associated multisystem inflammatory syndrome. Journal of the Pediatric Infectious Diseases Society, Chicago, 2020.

SOCIEDADE BRASILEIRA DE PEDIATRIA EM SÃO PAULO. Síndrome inflmatóra multissistêmica pediátrica (síndrome associada temporalmente ao COVID-19). São Paulo, 2020.

SOUZA, M. T.; SILVA, M. D.; CARVALHO, R. Revisão integrativa: o que é e como fazer. Einstein, São Paulo, v. 8, n. 1, p. 102-106, 2010.

WHO. Word Health Organization. Multisystem inflammatory syndrome in children and adolescents temporally related to COVID-19. [S. 1.], 2020.

\section{Como referenciar este artigo}

UCHIYA, Eduardo Haruo. Síndrome Inflamatória Pediátrica Multisistêmica (PIMS) e a associação com a SARS-CoV-2. Temas em Educ. e Saúde, Araraquara, v. 16, n. 1, p. 9-20, jan./jun., 2020. e-ISSN 2526-3471. DOI: https://doi.org/10.26673/tes.v16i1.13759

Submetido em: 05/04/2020

Revisões requeridas: $05 / 06 / 2020$

Aprovado em: 10/06/2020

Publicado em: 19/06/2020 